\title{
Xilitol na prevenção da Otite Média Aguda em crianças
}

Margarida Moreira, ${ }^{1}$ Margarida Ferreira da Silva, ${ }^{1}$ Francisco Pinto da Costa, ${ }^{1}$ Catarina Miranda ${ }^{2}$

\section{RESUMO}

Objetivo: A otite média aguda (OMA), patologia comum nas crianças, é responsável por uma percentagem significativa dos antibióticos prescritos em ambulatório. Contudo, a sua utilização é controversa, já que a doença pode recorrer durante a infância, levando a um excesso de utilização da antibioterapia e incremento das resistências bacterianas. O xilitol tem potencial na prevenção da OMA. O objetivo deste trabalho é rever a evidência disponível sobre a eficácia do xilitol na prevenção da OMA em crianças. Fontes de dados: National Guideline Clearinghouse, NHS Evidence, Canadian Medical Association Infobase, Scottish Intercollegiate Guidelines, Evidence Based Medicine, InfoPOEMs, TRIP Database, The Cochrane Library, DARE, Bandolier e Medline.

Métodos de revisão: Pesquisa de artigos publicados entre janeiro de 2000 e maio de 2012, usando os termos MeSH:xylitol e otitis media. Para avaliação dos níveis de evidência e atribuição de forças de recomendação foi utilizada a escala Strenght of Recommendation Taxonomy (SORT).

Resultados: Foram encontrados 106 artigos, dos quais três cumpriam os critérios de inclusão. As duas metanálises evidenciaram a eficácia do xilitol na redução da ocorrência de OMA em crianças (Nível de evidência 2); a revisão sistemática concluiu haver evidência de baixa qualidade para o uso profilático de xilitol na OMA (Nível de evidência 2).

Conclusões: A evidência disponível suporta a eficácia do xilitol na prevenção de OMA em crianças (SORT B). No futuro serão necessários mais estudos, multicêntricos, com maior follow-up, que clarifiquem a dose mais adequada de xilitol, os seus benefícios a longo prazo, a duração da intervenção e a população-alvo. Sugere-se, ainda, o desenvolvimento de outras vias de administração que permitam a melhor adesão possível em todas as faixas etárias.

Palavras-chave: Xilitol; Otite Média Aguda.

\section{INTRODUÇÃO}

A OMA é uma patologia muito frequente na infância, calculando-se que, entre as crianças comidade inferior a 3 anos, $83 \%$ já tenham tido pelo menos um episódio ${ }^{1}$ e cerca de $20 \%$ tenham pelo menos uma OMA por ano. ${ }^{2}$

Apesar de a sua etiologia ser variada e incluir mais frequentemente causas víricas, as causas bacterianas geram uma maior preocupação pela menor probabilidade de evolução espontânea favorável. ${ }^{3} \mathrm{~A}$ OMA bacteriana, se não tratada eficazmente, pode causar sequelas nomeadamente: otite média crónica, mastoidite, paralisia facial, labirintite e hipoacusia de condução que poderão condicionar, por sua vez, atrasos na linguagem, dificuldades de aprendizagem e de interação social, e, menos frequentemente, complicações graves como meningite e abcesso cerebral. ${ }^{3,4}$

${ }^{1}$ Internos de formação específica de Medicina Geral e Familiar na USF das Ondas, ACeS Grande Porto IV - Póvoa de Varzim/Vila do Conde

${ }^{2}$ Interna de formação específica de Medicina Geral e Familiar na USF do Mar, ACeS Grande Porto IV - Póvoa de Varzim/Vila do Conde
Esta entidade é responsável, nos países desenvolvidos, por uma percentagem significativa dos antibióticos prescritos em ambulatório. ${ }^{3}$ Nos Estados Unidos da América é a causa de metade das prescrições de antibióticos em crianças $^{5}$ e, como se sabe, esta sobreutilização de antibioterapia leva ao progressivo aparecimento de estirpes bacterianas resistentes. ${ }^{3}$ Além disso, a OMA associa-se a custos elevados, não só pela utilização de antibióticos, mas também pelo número de consultas médicas e pelo custo de tratamentos mais agressivos como cirurgias invasivas e hospitalizações para tratamento das complicações. ${ }^{6}$

Pelas razões mencionadas, justifica-se a necessidade de recurso a estratégias preventivas da OMA que sejam custo-efetivas, com efeitos positivos na qualidade de vida e com poucos efeitos laterais. Nesse sentido, várias abordagens são já usadas, como a adenoidectomia, a timpanostomia, a profilaxia antibiótica e o uso de imunoestimulantes e da vacina antipneumocócica que, apesar de tudo, têm uma eficácia limitada. ${ }^{7-10}$ Assim se entende a necessidade de se explorarem outras formas profiláticas, entre as quais o uso de xilitol parece ser promissor.

Oxilitol é um polissacarídeo, usado há muitos anos como 
adoçante por conter um terço das calorias da sacarose, preservando o mesmo sabor, tendo começado por ser usado em diabéticos, ${ }^{11}$ e que existe naturalmente em vegetais e frutas vermelhas como as ameixas, os morangos, as framboesas e as groselhas. ${ }^{12}$ Mais tarde, foi também adotado como adoçante nas pastilhas elásticas, substituindo outros açúcares, por ter um efeito anti-cariogénico demonstrado, ${ }^{13} \mathrm{com}$ a vantagem de ser, geralmente, bem tolerado e aceite pelas crianças e de apresentar efeitos laterais mínimos, nomeadamente apenas um discreto desconforto abdominal. ${ }^{14} \mathrm{Du}$ rante a investigação das suas potencialidades, também se veio a demonstrar, in vitro, o seu efeito na diminuição do crescimento do Streptococcus pneumoniae e a inibição da sua adesão às células da nasofaringe, ${ }^{15}$ mostrando que poderia ser uma substância promissora na prevenção da OMA ao ter este efeito naquele que é o seu principal agente bacteriano e que menor probabilidade tem de desaparecer espontaneamente do ouvido médio. ${ }^{3}$

O objetivo do nosso trabalho é rever a evidência disponível sobre a eficácia do xilitol na prevenção da OMA em crianças.

\section{MÉTODOS DE REVISÃO}

Foi realizada uma pesquisa de normas de orientação clínica, metanálises, revisões sistemáticas e ensaios clínicos aleatorizados e controlados (ECAC's) nas bases de dados $\mathrm{Me}$ dline, National Guideline Clearinghouse, NHS Evidence, Canadian Medical Association, Scottish Intercollegiate Guidelines, Evidence Based Medicine, InfoPOEMs, TRIP Database, The Cochrane Library, DARE e Bandolier, publicados entre janeiro de 2000 e maio de 2012, nas línguas portuguesa, inglesa e espanhola. Foram utilizados os termos Mesh: xylitol e otitis media.

Como critérios de inclusão consideraram-se os artigos cuja população fosse constituída por crianças até aos 12 anos, e em que a intervenção fosse o uso de xilitol comparativamente ao uso de placebo ou a ausência de intervenção. O resultado medido foi a ocorrência da OMA.

Foram usados como critérios de exclusão: artigos duplicados, artigos de opinião, artigos de revisão clássica de tema ou sumários de sites, ensaios clínicos incluídos em revisões sistemáticas mais recentes, discordância com o objetivo da revisão, artigos em que a população incluísse crianças não saudáveis (incluindo malformações craniofaciais, anomalias estruturais do ouvido médio e patologia aguda) ou medicadas com antibióticos por qualquer motivo.

Para avaliação dos níveis de evidência e atribuição de forças de recomendação foi utilizada a Escala SORT da American Academy of Family Physicians. ${ }^{16}$

\section{RESULTADOS}

Da pesquisa realizada resultaram 106 artigos, três dos quais cumpriam os critérios de inclusão: duas revisões sistemáticas com metanálise e uma revisão sistemática sem metanálise, cujas caraterísticas se resumem no Quadro I.

Segundo a metanálise da Cochrane de Azarpazhoohet al, ${ }^{6}$ que incluiu três ECAC's ${ }^{17,1,19}$ que compararam o uso do xilitol com o controlo em crianças saudáveis, o xilitol mostrou efeito na prevenção da ocorrência de OMA (RR 0,75; IC 95\%: 0,65-0,88). De acordo com uma subanálise deste artigo, as pastilhas elásticas poderão ser uma forma mais eficaz de administração em crianças que as possam mascar.

Danhauer et al, na sua metanálise, ${ }^{20}$ incluíram dois ECAC's ${ }^{17,18}$ que consideraram ser homogéneos e que demonstraram uma eficácia estatisticamente significativa do xilitol na redução da OMA comparativamente ao placebo (RR 0,68; IC 95\%: 0,57-0,83).

A revisão sistemática elaborada por Damoiseaux et al ${ }^{21} \mathrm{em}$ 2011 englobou três ECAC's, com uma amostra de 1826 crianças entre os 6 meses e os 7 anos. Verificou-se que estes três ECAC's são comuns a todos artigos de revisão encontrados, pelo que se procederá a uma descrição mais detalhada dos mesmos.

O estudo de Uhariet al, 1996, ${ }^{17}$ tratou-se de um ensaio clínico duplamente cego e aleatorizado, que teve lugar em crianças de uma região da Finlândia, entre os 2 e os 5 anos. Este comparou a utilização de duas pastilhas elásticas, cinco vezes ao dia, com xilitol 8,4 g/dia ( $\mathrm{n}=157$ ), com pastilhas elásticas placebo (n=149), após refeições, quanto à ocorrência de OMA ao longo de 2 meses. Os resultados respeitantes aos 306 participantes mostraram uma redução do risco absoluto (RRA) de OMA estatisticamente significativa $(0,087$; IC 95\%: 0,004-0,17), com o uso de xilitol. Não foram relatados efeitos laterais.

Um segundo ECAC de Uhari et al, 1998, ${ }^{18}$ envolveu 857 crianças da mesma região do estudo anteriormente descrito, entre os 6 meses e os 7 anos. A distribuição foi realizada segundo dois grupos: as capazes e as não capazes de mascar pastilha elástica. Dentro do primeiro, as crianças foram aleatorizadas para um de três ramos: pastilha elástica com xilitol, duas pastilhas, 5 vezes ao dia, equivalendo a $8,4 \mathrm{~g}$ de xilitol ( $\mathrm{n}=179)$ ou pastilha elástica de controlo $(\mathrm{n}=178)$ ou losangos com xilitol, 3 peças, 5 vezes ao dia, numa dose de 10 $\mathrm{g} /$ dia $(\mathrm{n}=176)$. No segundo, as crianças foram distribuídas aleatoriamente para receber: xarope de xilitol $400 \mathrm{~g} / \mathrm{L}, 5 \mathrm{~mL}$, 5 vezes por dia, perfazendo $10 \mathrm{~g}$ de xilitol por dia $(\mathrm{n}=159)$ ou xarope de controlo $(n=165)$. A administração era feita sempre após uma refeição e o ensaio decorreu ao longo de 3 meses. Adupla ocultação verificou-se em relação às crianças que 
recebiam pastilhas elásticas e às que recebiam xarope. Em relação aos resultados, as desistências foram superiores nos ramos do xarope de xilitol e dos losangos, onde também foram reportadas mais queixas de desconforto abdominal, tendo sido realizada uma análise intention-to-treat. Assim, a análise pastilhas de xilitol $v s$. pastilhas de controlo mostrou que as primeiras se associavam a uma redução estatisticamente significativa da ocorrência de OMA de 0,65 episó-

\begin{tabular}{|c|c|c|c|c|c|}
\hline Referência & Ensaios incluídos & Intervenção & Resultados & Conclusão & $\begin{array}{l}\text { Nível de } \\
\text { evidência }\end{array}$ \\
\hline $\begin{array}{l}\text { Azarpazhooh et } \\
\text { al, }{ }^{6} 2011 \\
\text { Cochrane } \\
\text { Reviews } \\
\text { Metanálise }\end{array}$ & $\begin{array}{l}\text { - até agosto } 2011 \\
\text { - } 3 \text { ECAC } \\
\text { - } \mathrm{n}=1826 \\
\text { - } 6 \text { meses- } 7 \text { anos } \\
\text { - follow-up: } \\
\text { 2-3 meses }\end{array}$ & $\begin{array}{l}\text { Xilitol (pastilhas } \\
\text { elásticas, losangos - } 8,4 \\
\text { a } 10 \mathrm{~g} / \mathrm{dia} \text { ou xarope } \\
400 \mathrm{~g} / \mathrm{L}-10 \mathrm{~g} / \mathrm{dia} \text {, } \\
3 \text { a } 5 \mathrm{x} / \mathrm{dia} \text { ) } \\
\text { vs. } \\
\text { Placebo (pastilhas } \\
\text { elásticas ou xarope) }\end{array}$ & $\begin{array}{l}\text { RR 0,75 } \\
\text { (IC 95\%: 0,65-0,88), } \\
P<0,01\end{array}$ & $\begin{array}{l}\text { Xilitol reduz } \\
\text { ocorrência } \\
\text { de OMA }\end{array}$ & 2 \\
\hline $\begin{array}{l}\text { Danhauer et } \\
a^{20} 2010 \\
\text { Metanálise }\end{array}$ & $\begin{array}{l}-2 \text { ECAC } \\
-\mathrm{n}=1193 \\
-6 \text { meses }-6 \text { anos } \\
\text { - follow-up: } 2-3 \\
\text { meses }\end{array}$ & $\begin{array}{l}\text { Xilitol (pastilhas } \\
\text { elásticas, losangos } \\
-8,4 \mathrm{~g} / \text { dia ou xarope } \\
400 \mathrm{~g} / \mathrm{L}-10 \mathrm{~g} / \mathrm{dia} \text {, } \\
5 \mathrm{x} / \mathrm{dia} \text { ) vs. } \\
\text { Placebo (pastilhas } \\
\text { elásticas ou xarope) }\end{array}$ & $\begin{array}{l}\text { RR: 0,68 } \\
\text { (IC 95\%: 0,57-0,83), } \\
P<0,0001\end{array}$ & $\begin{array}{l}\text { Xilitol reduz } \\
\text { ocorrência } \\
\text { de OMA }\end{array}$ & 2 \\
\hline $\begin{array}{l}\text { Damoiseaux et } \\
a l^{21} 2011 \\
\text { Revisão } \\
\text { sistemática }\end{array}$ & $\begin{array}{l}\text { - até setembro } \\
2010 \\
\text { - } 3 \text { ECAC } \\
\text { - } 6 \text { meses - } 7 \\
\text { anos } \\
\text { - follow-up: } 2-3 \\
\text { meses }\end{array}$ & $\begin{array}{l}\text { Xilitol } \\
\text { vs. } \\
\text { Placebo }\end{array}$ & 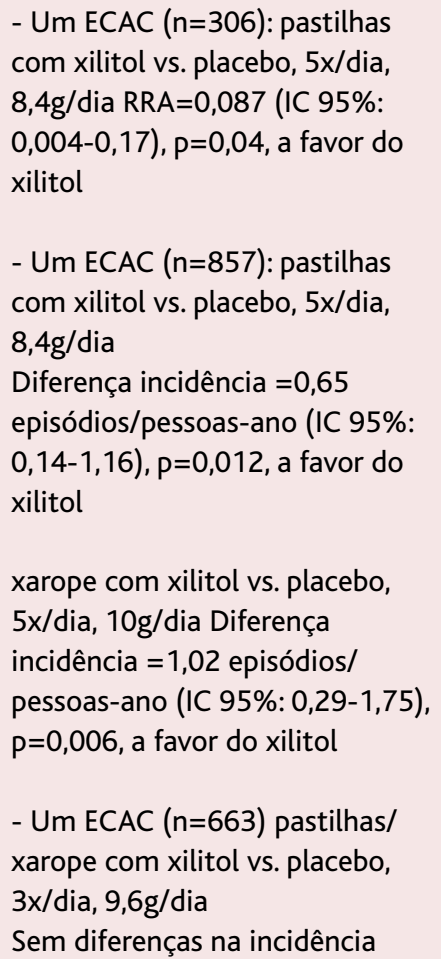 & $\begin{array}{l}\text { Evidência } \\
\text { com pouca } \\
\text { consistência } \\
\text { de que o } \\
\text { xilitol reduz } \\
\text { a ocorrência } \\
\text { da OMA }\end{array}$ & 2 \\
\hline
\end{tabular}

RR - Risco relativo; RRA - Redução do risco absoluto. 
dios/pessoas-ano (IC95\%: 0,14-1,16, p=0,012). O mesmo não aconteceu com o uso de losangos, que não foi eficaz. Por outro lado, observou-se uma diferença na incidência de 1,02 episódios/pessoas-ano entre aquelas que receberam xarope de controlo e as que receberam xarope de xilitol, a favor deste último (IC95\%: 0,29-1,75, p=0,006). Deste modo, este estudo concluiu que, nas crianças que não são capazes de mascar pastilhas elásticas, o xarope de xilitol é uma forma eficaz de prevenção da OMA, sendo que, nas restantes, as pastilhas elásticas com xilitol também o são.

Por último, um outro estudo, ${ }^{19}$ com dupla ocultação e uma duração de 3 meses, compreendeu 663 crianças entre os 7 meses e os 7 anos, que foram aleatorizadas para um de dois grupos: o que recebeu produto com xilitol numa dose diária de 9,6g - 2 pastilhas elásticas ou $8 \mathrm{~mL}$ de xarope de xilitol $400 \mathrm{~g} / \mathrm{L}$ ( $\mathrm{n}=332$ ) e o de controlo ( $\mathrm{n}=331$ ), recebendo a intervenção dividida em 3 doses diárias, após refeições. Nas crianças que não eram capazes de mascar pastilha elástica, optou-se por fazer a administração sob a forma de xarope. O ensaio verificou não haver diferenças estatisticamente significativas entre os dois grupos em relação à incidência de OMA, ou seja, que a posologia do xilitol em 3 doses diárias não é eficaz. Não foram relatados efeitos laterais.

\section{CONCLUSÕES}

Considera-se, assim, que existe evidência do benefício do uso de xilitol na prevenção da OMA em crianças saudáveis, que deriva de duas metanálises e uma revisão sistemática com nível de evidência 2 , uma vez que os ensaios clínicos em que se basearam apresentam alguns resultados inconsistentes. Por este motivo, foi atribuída uma força de recomendação B.

Contudo, deve-se ter em consideração a existência de algumas limitações dos estudos, pois estes apresentam amostras pequenas, da mesma área geográfica, com idade média dos participantes superior ao pico de incidência da OMA (612 meses), ${ }^{22}$ seguidas por curtos intervalos de tempo (2-3 meses) e utilizadas como base das três revisões. O curto período de seguimento não permite antever efeitos a longo prazo ou a duração-alvo da utilização de xilitol para obtenção dos efeitos pretendidos. Além disso, utilizaram-se diferentes dosagens e vias de administração, nem todas com a mesma eficácia. Oxilitol, através de pastilhas elásticas ou xarope, é fornecido, de forma eficaz, cinco vezes ao dia, o que, apesar da elevada adesão nos estudos, pode dificultar a sua implementação, sendo necessário avaliar novas formulações. Nenhum dos estudos fez uma subanálise das crianças com fatores de risco possíveis para a ocorrência de OMA, como o consumo de tabaco pelos pais, o uso de chupeta, a existência de familiares com maior suscetibilidade a OMA e o estado de portador para pneumococos. Da mesma forma, não fizeram subanálises em grupos de crianças com fatores de proteção para a mesma, como a amamentação ou o recurso prévio a outras formas de profilaxia da OMA.

Desta forma, no futuro serão necessários novos estudos, multicêntricos, com mais participantes e um follow-up mais longo, que clarifiquem: vias de administração em crianças mais pequenas e que não podem mascar pastilhas elásticas; doses mais convenientes, eventualmente mais baixas, ou fornecidas com uma frequência mais cómoda; a duração-alvo da administração do xilitol; os efeitos profiláticos do xilitol em crianças com maior risco de OMA; o papel do xilitol na prevenção de determinadas complicações da OMA; a diferença do benefício do xilitol usado isoladamente ou como parte de uma estratégia multifatorial de prevenção da OMA; e, por fim, os efeitos laterais exatos do xilitol nas doses recomendadas. É de referir, ainda, que a introdução de uma medida deste género requer também a aceitação e conhecimento de médicos, pais e cuidadores que lidam diariamente com a criança.

Esta revisão conclui que há evidência de que o xilitol reduz o risco de ocorrência de OMA em crianças entre os 6 meses e os 7 anos, se fornecido através de pastilhas elásticas, mascadas até não ter sabor ou durante 5 minutos, na quantidade de 8,4 g/dia (duas pastilhas elásticas, cinco vezes por dia), ou xarope na concentração $400 \mathrm{~g} / \mathrm{L}, 10 \mathrm{~g} / \mathrm{dia}$, cinco vezes por dia, após as refeições ou snacks. Esta estratégia revela-se promissora, sobretudo em áreas com acesso limitado a cuidados de saúde. No entanto, torna-se limitada a sua aplicação em crianças mais jovens, uma vez que o xarope de xilitol não se encontra comercializado em Portugal. São também necessários novos estudos antes de se difundir a aplicação desta medida.

\section{AGRADECIMENTOS}

À Dra. Carla Morna pela disponibilidade mostrada sempre.

\section{REFERÊNCIAS BIBLIOGRÁFICAS}

1. Teele DW, Klein JO, Rosner B. Epidemiology of otitis media during the first seven years of life in children in greater Boston: a prospective, cohort study. J Infect Dis 1989 Jul; 160 (1): 83-94.

2. Carvalho IP, Silva MC, orgs. Urgência Pediátrica Integrada do Porto: Orientações clínicas - Ambulatório em idade pediátrica. Porto:ARS Norte; 2008. p. 835.

3. Sassetti L, coord. Urgências no ambulatório em Idade Pediátrica, volume II. Orientações Técnicas 14. Lisboa: Direção-Geral de Saúde; 2005. p. 11-3.

4. Rosenfeld RM, Goldsmith AJ, Tetlus L, Balzano A. Quality of life for children with otitis media. Arch Otolaryngol Head Neck Surg 1997 Oct; 123 (10): 1049-54.

5. American Academy of Pediatrics Subcommittee on Management of Acute Otitis Media. Clinical practice guideline: diagnosis and management of acute otitis media. Pediatrics 2004 May; 113 (5): 1451-65. 
6. Azarpazhooh A, Limeback H, Lawrence HP, Shah PS. Xylitol for preventing acute otitis media in children up to 12 years of age (Review). Cochrane Database Syst Rev 2011 Nov 9; (11): CD007095.

7. Arason VA, Kristinsson KG, Sigurdsson JA, Stefánsdóttir G, Mölstad S, Gudmundsson S. Do antimicrobials increase the carriage rate of penicillin resistant pneumococci in children? Cross sectional prevalence study. BMJ 1996 Aug 17; 313 (7054): 387-91.

8. Paradise JL, Bluestone CD, Colborn DK, Bernard BS, Smith CG, Rockette HE, et al. Adenoidectomy and adenotonsillectomy for recurrent acute otitis media: parallel randomized trials in children not previously treated with tympanostomy tubes. JAMA 1999 Sep 8; 282 (10): 945-53.

9. Eskola J, Kilpi T, Palmu A, Jokinen J, Haapakoski J, Herva E, et al. Efficacy of a pneumococcal conjugate vaccine against acute otitis media. N Engl J Med 2001 Feb 8; 344 (6): 403-9.

10. Williams RL, Chalmers TC, Stange KC, Chalmers FT, Bowlin SJ. Use of antibiotics in preventing recurrent acute otitis media and in treating otitis media with effusion. A meta-analytic attempt to resolve the brouhaha. JAMA $1993 \mathrm{Sep}$ 15; 270 (11): 1344-51.

11. Milgrom P, Rothen M, Milgrom L. Developing public health interventions with xylitol for the US and US-associated territories and states. Suom Hammaslaakarilehti 2006 May 15; 13 (10-11): 2-11.

12. Mäkinen KK, Söderling E.A quantitative study of mannitol, sorbitol, xylitol, and xylose in wild berries and commercial fruits. J Food Sci 1980 Mar; 45 (2): 36771.

13. Hildebrandt G, Lee IK. Xylitol containing oral products for preventing dental caries [protocol]. Cochrane Database Syst Rev 2004; (1): CD004620.

14. Akerblom HK, Koivukangas T, Puukka R, Mononen M. The tolerance of increasing amounts of dietary xylitol in children. Int J Vitam Nutr Res Suppl 1982 22: $53-66$.

15. Kontiokari R, Uhari M, Koskela M. Effect of xylitol on growth of nasopharyngeal bacteria in vitro. Antimicrob Chemother 1995 Aug; 39 (8): 1820-3.

16. Ebell MH, Siwek J, Weiss BD, Woolf SH, Susman J, Ewigman B, et al. Strength of Recommendation Taxonomy (SORT): a patient-centered approach to grading evidence in the medical literature. Am Fam Physician 2004 Feb 1; 69 (3): 548-56.

17. Uhari M, Kontiokari T, Koskela M. Niemelä M. Xylitol chewing gum in prevention of acute otitis media: double blind randomised trial. BMJ 1996 Nov 9; 313 (7066): 1180-4.

18. Uhari M, Kontiokari T, Niemelä M. A novel use of xylitol sugar in preventing acute otitis media. Pediatrics 1998 Oct; 102 (4 Pt 1) :879-84.

19. Hautalahti O, Renko M, Tapiainen T, Kontiokari T, Pokka T, Uhari M. Failure of xylitol given three times a day for preventing acute otitis media. Pediatr Infect Dis J 2007 May; 26 (5): 423-7.

20. Danhauer JL, Johnson CE, Corbin NE, Bruccheri KG. Xylitol as a prophylaxis for acute otitis media: systematic review. Int J Audiol 2010 Oct; 49 (10): 754-61.

21. Damoiseaux RA, Rovers MM. AOM in children. Clin Evid (Online) 2011 May 10; 2011. pii 0301.

22. Advisory Committee on Immunization Practices. Preventing pneumococcal disease among infants and young children. Recommendations of the Advisory Committee on Immunization Practices (ACIP). MMWR Recomm Rep 2000 Oct 6; 49 (RR-9): 1-35.

\section{CONFLITOS DE INTERESSE}

Os autores declaram não ter conflito de interesses.

\section{ENDEREÇO PARA CORRESPONDÊNCIA}

Margarida Moreira

Rua da Praia, n. ${ }^{\circ} 186$ - Fieiro - Aguçadoura, 4495-031 Póvoa de Varzim

E-mail:mmargmoreira@gmail.com

Recebido em 15-04-2013

Aceite para publicação em 17-12-2013

Artigo escrito ao abrigo do novo acordo ortográfico.

\section{ABSTRACT}

\section{XYLITOL FOR PREVENTION OF ACUTE OTITIS MEDIA IN CHILDREN}

Aim: Acute otitis media (AOM) is a common illness in children and accounts for a significant proportion of antibiotic prescriptions. However antibiotic use is controversial since the disease may be self-limiting and recurrent in childhood and excessive antibiotic use may lead to increased bacterial resistance. Xylitol has potential in preventing AOM. This review examines the evidence for the effectiveness of xylitol in preventing AOM in children.

Data sources: National Guideline Clearinghouse, NHS Evidence, Canadian Medical Association, Scottish Intercollegiate Guidelines, Evidence Based Medicine, Info POEMs, TRIP, The Cochrane Library, DARE, Bandolier and Medline.

Review methods: A search was performed between January 2000 and May 2012, in Portuguese, English and Spanish, using otitis media and xylitol as MeSH terms. The Strength of Recommendation Taxonomy (SORT) scale was used to assess level of evidence and strength of recommendations.

Results: Of the 106 articles found, 3 met the inclusion criteria. Two meta-analyses found evidence for the efficacy of xylitol in reducing the occurrence of AOM in children (Level of Evidence 2). A systematic review concluded that there was poor quality evidence for the use of xylitol in preventing AOM (Level of Evidence 2).

Conclusions: Current evidence shows the efficacy of xylitol in preventing AOM in children (SORT B). Questions remain regarding the dose of xylitol, its long-term benefits, the duration of treatment, and the target population. Research on routes of administration providing better adherence to treatment in all age groups is needed.

Key-words: Xylitol; Acute Otitis Media. 